\title{
Effects of Tungsten and Molybdenum on Sucrose Content and Hydrolytic Enzymes of Immature Sugarcane ${ }^{1}$
}

\author{
Alex G. Alexander'
}

\section{INTRODUCTION}

Molybdenum has been shown to inhibit phosphatase in immature sugarcane $(2)^{3}$, with increased sucrose forming as an apparent side-effect. More recent studies revealed that $M$ lo suppressed amylase as well as phosphatase (8), although no amylase-Mo relationship has been found in vitro (7) ${ }^{4}$. However, other in vitro studies have shown that $\mathrm{W}$ is at least as effective as Mo in suppressing phosphatase (5). The prospect thus arises that W might serve as an effective enzyme inhibitor in living cane.

Experiments were set up in which both $M o$ and $W$ were supplied to immature sugarcane as foliar sprays. There were three objectives: 1 , Verification of Mo-phosphatase-sucrose relationships; 2, to determine whether $\mathrm{W}$ would likewise inhibit phosphatase, and amylase, or possibly invertase, which is also a hydrolytic catalyst; and 3, to learn whether $\mathrm{W}$ can promote sucrose production as a consequence of enzyme inhibition.

\section{MATERIALS AND METHODS}

Sugarcane of the variety M. 336 was grown in sand culture, and treated, and harvested in accordance with procedures described previously (8). Molybdenum, at rates of $10,20,40$, and 80 p.p.m., and W at rates of 1,10 , 100 , and 200 p.p.m., were applied as foliar sprays when the plants were 12 weeks of age. Control plants received distilled water with wetting agent. The experimental design was a completely randomized block with three replicates for each of the nine treatments.

Leaf and meristem samples were harvested a few moments prior to treatment, and at 3, 9, and 27 days thereafter. All samples were frozen and prepared for extraction as described in the earlier report (8).

${ }^{1}$ For convenience, the following symbols and abbreviations are used throughout this paper: Tungsten, (W); molybdenum, (Mo); parts per million (p.p.m.); $\beta$-glycerophosphate ( $\beta$-GP); and glucose-1-phosphate (G-1-P).

${ }^{2}$ Associate Plant Physiologist, Agricultural Experiment Station, University of Puerto Rico, Río Piedras, P.R.

${ }^{3}$ Italic numbers in parentheses refer to Literature Cited, pp. 441-2.

'Suppressed amylase has heen found associated with sucrose-promoting treatments $(I, S)$. Attempts to increase sucrose by use of amylase inhibitors had been contemplated, but not attempted, prior to the present experiments. 
Sugars and enzyme protein were extracted from the freeze-dried tissues with distilled water. Suspensions of leaf material were treated by sonic disintegration prior to extraction, whereas meristem samples were extracted directly. Sugar assays were conducted with clarified water extracts. Total ketose was determined colorimetrically by the resorcinol method of Roe (10), and sucrose by the modification of Cardini et al. (9), which destroys fructose by heating the samples for 10 minutes at $100^{\circ} \mathrm{C}$. in $0.25 \mathrm{~N} \mathrm{NaOH}$. Fructose was estimated by subtracting sucrose values from total ketose. Enzyme protein was precipitated, dialyzed, and measured as described earlier (8).

Enzymes assayed during the present studies included amylase, invertase, and the acid phosphatases which hydrolyze $\beta$-GP, ATP, and G-1-P. Phosphatase procedures were described previously (1), as were those for amylase (7) and invertase (6).

All data collected during these investigations were subjected to statistical analysis of variance, and means were compared by the StudentNewman-Keuls Q Test. Results of the statistical analyses are summarized in table 1.

\section{RESULTS AND DISCUSSION}

SUCROSE CHANGES IN RESPONSE TO TUNGSTEN AND MOLYBDENUM

Both Mo and W suppressed enzymes and increased sucrose during the present study, with Mo apparently more effective as an enzyme inhibitor, and $\mathrm{W}$ causing the greater sucrose increases. However, treatments were not the major source of variation.

Leaf sugars for both treatment and control plants declined moderately at 3 and 9 days following treatment, and experienced a sharp decline at 27 days (table 2). Meristem sugar levels were more stable, but nevertheless a pronounced sucrose decline was recorded at 9 days. Leaf enzyme activity was highest at 9 days and declined severely by 27 days (table 3). Meristem enzymes also evidenced decline at 27 days. These changes in sugar and enzyme levels undoubtedly reflect vigorous growth and biochemical activity in the young plants, for which sucrose production was not likely a major consideration. Treatment effects were secondary against this background. Nevertheless, definite $M o$ and $W$ effects did occur.

Tungsten at 10 p.p.m. was the most effective treatment of the study for increasing sucrose in both leaf and meristem (table 2, items $3,12,21$, and 30 ). At 3 days, the 1-p.p.m. W treatment nearly doubled sucrose levels in meristem, and caused a moderate increase of leaf sucrose. Nearly all levels of $W$ appeared to increase meristem sucrose at all posttreatment harvests, with the lone exception being 1 p.p.m.W at 27 days. Two hundred parts per million of $\mathrm{W}$ increased leaf sucrose at 3 and 27 days. The reader should 
TABLE 1.-Summary of significant mean differences among sugar and enzyme values of immature sugarcane in response to control, $W$, and Mo treatments'

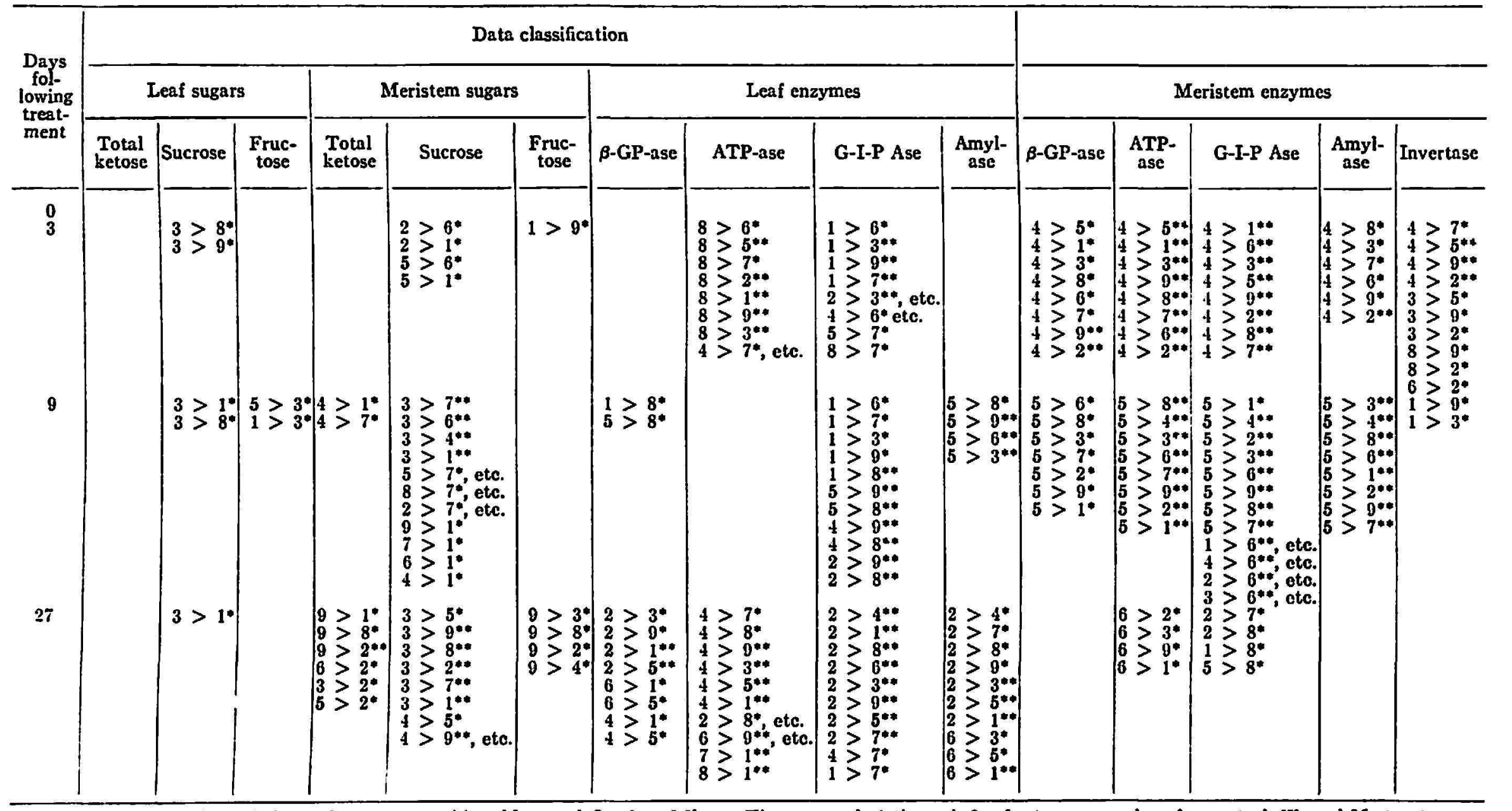

1 The numbers and symbols used to prepare this table are defined as follows: The numerals 1 through 9 refer to mean values for control, $W$, and Mo treatments. Number $1=$ control; $2=W, 1$ p.p.m.; $3=W, 10$ p.p.m.; $4=W, 100$ p.p.m.; $5=W, 200$ p.p.m.; $6=$ MIo, 10 p.p.m.; $7=$ Mo, 20 p.p.m.; $8=M 10,40$ p.p.m.; and $9=$ Mo, 80 p.p.m. The symbol > appearing between 2 figures indicates that the first mean was of greater magnitude than the second. One asterisk denotes significant mean of descending value and after recording the first significant term, all subsequent values are accompanied by "etc." rather than rewrite the remaining series of means. 
TaBle 2.-Mean values for leaf and meristem sugars of immalure sugarcane following foliar application of tungsten and molybdenum ${ }^{1}$

\begin{tabular}{|c|c|c|c|c|c|c|c|c|}
\hline \multirow{3}{*}{$\begin{array}{c}\text { Days } \\
\text { follow- } \\
\text { ing } \\
\text { treat- } \\
\text { ment }\end{array}$} & \multirow{3}{*}{$\begin{array}{l}\text { Item } \\
\text { No. }\end{array}$} & \multirow{3}{*}{ Treatment } & \multicolumn{6}{|c|}{ Data classification } \\
\hline & & & \multicolumn{3}{|c|}{$\begin{array}{c}\text { Leaf sugars } \\
\text { (mg./gm. dry weight) }\end{array}$} & \multicolumn{3}{|c|}{$\begin{array}{c}\text { Mieristem sugars } \\
\text { (mg./gm. dry weight) }\end{array}$} \\
\hline & & & $\underset{\text { ketose }}{\text { Total }}$ & Sucrose & Fructose & $\begin{array}{c}\text { Total } \\
\text { ketose }\end{array}$ & Sucrose & Fructose \\
\hline \multirow[t]{4}{*}{0} & $\begin{array}{l}1 \\
2 \\
3 \\
4 \\
5\end{array}$ & $\begin{array}{l}\text { Control } \\
\text { W, } 1 \text { p.p.m. } \\
\text { W, } 10 \text { p.p.m. } \\
\text { W, } 100 \text { p.p.m. } \\
\text { W, } 200 \text { p.p.m. }\end{array}$ & $\begin{array}{r}115.2 \\
109.9 \\
116.0 \\
112.7 \\
99.4\end{array}$ & $\begin{array}{l}63.9 \\
69.4 \\
71.5 \\
70.7 \\
61.1\end{array}$ & $\begin{array}{l}51.3 \\
40.5 \\
44.5 \\
42.0 \\
38.5\end{array}$ & $\begin{array}{l}158.0 \\
166.0 \\
166.8 \\
170.3 \\
172.7\end{array}$ & $\begin{array}{l}27.6 \\
34.5 \\
36.8 \\
36.4 \\
30.1\end{array}$ & $\begin{array}{l}130.1 \\
131.5 \\
130.0 \\
133.9 \\
142.5\end{array}$ \\
\hline & & W mean & 109.3 & 68.4 & 41.4 & 168.9 & 34.5 & 134.2 \\
\hline & $\begin{array}{l}6 \\
7 \\
8 \\
9\end{array}$ & $\begin{array}{l}\text { Mo, } 10 \text { p.p.m. } \\
\text { Mo, } 20 \text { p.p.m. } \\
\text { Mo, } 40 \text { p.p.m. } \\
\text { Mo, } 80 \text { p.p.m. }\end{array}$ & $\begin{array}{l}112.7 \\
108.8 \\
116.4 \\
116.4\end{array}$ & $\begin{array}{l}57.5 \\
72.8 \\
69.4 \\
69.3\end{array}$ & $\begin{array}{l}55.2 \\
36.0 \\
45.5 \\
47.1\end{array}$ & $\begin{array}{l}157.5 \\
136.8 \\
154.5 \\
166.0\end{array}$ & $\begin{array}{l}26.3 \\
25.3 \\
29.5 \\
34.7\end{array}$ & $\begin{array}{l}131.2 \\
131.2 \\
125.1 \\
131.3\end{array}$ \\
\hline & & Mo mean & 113.6 & 67.3 & 45.9 & 153.7 & 28.9 & 129.7 \\
\hline \multirow[t]{4}{*}{3} & $\begin{array}{l}10 \\
11 \\
12 \\
13 \\
14\end{array}$ & $\begin{array}{l}\text { Control } \\
\text { W, } 1 \text { p.p.m. } \\
\text { W, } 10 \text { p.p.m. } \\
\text { W, } 100 \text { p.p.m. } \\
\text { w, } 200 \text { p.p.m. }\end{array}$ & $\begin{array}{r}98.8 \\
108.3 \\
103.8 \\
89.7 \\
98.9\end{array}$ & $\begin{array}{l}51.3 \\
62.8 \\
70.6 \\
51.5 \\
62.0\end{array}$ & $\begin{array}{l}47.5 \\
45.4 \\
33.1 \\
38.3 \\
36.9\end{array}$ & $\begin{array}{l}159.9 \\
150.7 \\
160.9 \\
157.3 \\
154.7\end{array}$ & $\begin{array}{l}24.8 \\
47.6 \\
33.3 \\
41.6 \\
44.8\end{array}$ & $\begin{array}{l}134.2 \\
103.1 \\
127.6 \\
115.7 \\
109.9\end{array}$ \\
\hline & & W mean & 100.2 & 61.7 & 35.9 & 155.9 & 41.8 & 114.1 \\
\hline & $\begin{array}{l}15 \\
16 \\
17 \\
18\end{array}$ & $\begin{array}{l}\text { Mo, } 10 \text { p.p.m. } \\
\text { Mo, } 20 \text { p.p.m. } \\
\text { Mo, } 40 \text { p.p.m. } \\
\text { Mo, } 80 \text { p.p.m. }\end{array}$ & $\begin{array}{l}94.4 \\
85.7 \\
93.8 \\
82.1\end{array}$ & $\begin{array}{l}51.6 \\
54.1 \\
51.0 \\
46.3\end{array}$ & $\begin{array}{l}42.8 \\
31.7 \\
42.8 \\
35.8\end{array}$ & $\begin{array}{l}157.6 \\
157.3 \\
162.3 \\
142.7\end{array}$ & $\begin{array}{l}25.0 \\
38.1 \\
29.9 \\
41.5\end{array}$ & $\begin{array}{l}132.2 \\
129.5 \\
132.4 \\
101.2\end{array}$ \\
\hline & & Mo mean & 89.0 & 50.7 & 38.3 & 154.9 & 33.6 & 123.8 \\
\hline \multirow[t]{4}{*}{9} & $\begin{array}{l}19 \\
20 \\
21 \\
22 \\
23\end{array}$ & $\begin{array}{l}\text { Control } \\
\text { w, } 1 \text { p.p.m. } \\
\text { w, } 10 \text { p.p.m. } \\
\text { w, } 100 \text { p.p.m. } \\
\text { w, } 200 \text { p.p.m. }\end{array}$ & $\begin{array}{l}108.5 \\
109.3 \\
105.3 \\
113.1 \\
111.1\end{array}$ & $\begin{array}{l}56.9 \\
72.1 \\
80.3 \\
63.3 \\
63.3 \\
\end{array}$ & $\begin{array}{l}51.6 \\
37.2 \\
24.0 \\
49.7 \\
53.1\end{array}$ & $\begin{array}{l}138.9 \\
156.0 \\
157.6 \\
162.7 \\
145.8\end{array}$ & $\begin{array}{l}13.3 \\
35.3 \\
40.4 \\
21.8 \\
35.5\end{array}$ & $\begin{array}{l}125.6 \\
120.7 \\
117.2 \\
140.8 \\
110.4\end{array}$ \\
\hline & & $\mathrm{W}$ mean & 109.9 & 69.8 & 41.0 & 155.3 & 33.2 & 122.3 \\
\hline & $\begin{array}{l}24 \\
25 \\
26 \\
27\end{array}$ & $\begin{array}{l}\text { Mo, } 10 \text { p.p.m. } \\
\text { Mo, } 20 \text { p.p.m. } \\
\text { Mo, } 40 \text { p.p.m. } \\
\text { Mo, } 80 \text { p.p.m. }\end{array}$ & $\begin{array}{l}103.8 \\
112.2 \\
101.0 \\
106.8\end{array}$ & $\begin{array}{l}58.6 \\
63.3 \\
60.9 \\
66.9\end{array}$ & $\begin{array}{l}45.2 \\
50.4 \\
40.1 \\
39.9\end{array}$ & $\begin{array}{l}141.6 \\
138.1 \\
144.0 \\
144.1\end{array}$ & $\begin{array}{l}23.6 \\
24.7 \\
35.5 \\
30.8\end{array}$ & $\begin{array}{r}102.1 \\
96.0 \\
99.6 \\
94.4\end{array}$ \\
\hline & & Mo mean & 105.9 & 62.4 & 43.9 & 141.9 & 28.7 & 98.0 \\
\hline
\end{tabular}


TABLE 2.-Conlinued

\begin{tabular}{|c|c|c|c|c|c|c|c|c|}
\hline \multirow{3}{*}{$\begin{array}{l}\text { Days } \\
\text { follow- } \\
\text { ing } \\
\text { treat- } \\
\text { ment }\end{array}$} & \multirow{3}{*}{$\begin{array}{l}\text { Item } \\
\text { no. }\end{array}$} & \multirow{3}{*}{ Treatment } & \multicolumn{6}{|c|}{ Data classification } \\
\hline & & & \multicolumn{3}{|c|}{$\begin{array}{l}\text { Leaf sugars } \\
\text { (mg./gm. dry weight) }\end{array}$} & \multicolumn{3}{|c|}{$\begin{array}{c}\text { Meristem sugars } \\
\text { (mg./gm. dry weight) }\end{array}$} \\
\hline & & & $\begin{array}{c}\text { Total } \\
\text { ketose }\end{array}$ & Sucrose & Fructose & $\begin{array}{l}\text { Total } \\
\text { ketose }\end{array}$ & Sucrose & Fructose \\
\hline \multirow[t]{11}{*}{27} & 28 & Control & 55.1 & 31.0 & 24.1 & 135.4 & 33.3 & 102.1 \\
\hline & 29 & W, 1 p.p.m. & 56.2 & 36.6 & 20.9 & 130.7 & 34.7 & 96.0 \\
\hline & 30 & w, 10 p.p.m. & 77.7 & 44.9 & 32.7 & 147.7 & 48.1 & 99.6 \\
\hline & 31 & W, 100 p.p.m. & 57.3 & 36.4 & 20.9 & 140.8 & 46.4 & 94.4 \\
\hline & 32 & W, 200 p.p.m. & 57.3 & 41.4 & 15.9 & 146.9 & 39.5 & 107.5 \\
\hline & & W mean & 62.1 & 39.8 & 22.6 & 141.5 & 42.2 & 99.4 \\
\hline & 33 & Mo, 10 p.p.m. & 57.2 & 32.2 & 25.0 & 148.8 & 41.5 & 107.3 \\
\hline & 34 & Mo, 20 p.p.m. & 55.2 & 31.3 & 23.9 & 140.7 & 33.5 & 107.2 \\
\hline & 35 & Mo, 40 p.p.m. & 52.3 & 31.5 & 20.9 & 134.0 & 35.1 & 98.9 \\
\hline & 36 & Mo, 80 p.p.m. & 56.6 & 34.3 & 22.3 & 152.4 & 36.1 & 116.3 \\
\hline & & Mo mean & 55.3 & 32.3 & 23.0 & 143.9 & 36.5 & 107.4 \\
\hline \multirow{4}{*}{\multicolumn{2}{|c|}{$\begin{array}{l}\text { Grand meall } \\
\text { for: }\end{array}$}} & & & & & & & \\
\hline & & Control & 94.4 & 50.8 & 43.6 & 147.8 & 24.8 & 123.0 \\
\hline & & & 95.4 & 59.9 & 35.2 & 155.4 & 37.9 & 117.5 \\
\hline & & Mo & 91.0 & 53.2 & 37.8 & 148.6 & 31.9 & 114.7 \\
\hline
\end{tabular}

1 Each figure represents the computed mean of 3 replicates.

note, however, that many plants already contained more sucrose than the controls before treatments were applied.

Twenty parts per million of Mo caused slight increases of leaf sucrose at 3 and 9 days, but this was in no way comparable to that induced by $W$, nor to earlier results obtained with Mo (2,8). Molybdenum was more effective in promoting meristem sucrose, but here again results were not consistent among treatments. As was true of $\mathrm{W}$, all Mo treatments appeared to increase meristem sucrose at 9 days. This may be interpreted as a failure of treated plants to experience a sucrose decline comparable to that of controls.

\section{ENZYME CHANGES IN RESPONSE TO TUNGSTEN AND MOLYBDENUM}

Both $\mathrm{Mo}$ and $\mathrm{W}$ are confirmed phosphatase inhibitors when acting in vitro (5), but only G-1-P ase was inhibited by these elements during the present study (tables 3 and 4). On the contrary, ATP-ase was at times 
TABLE 3.-Mean values for leaf enzymes of immature sugarcane following foliar applicalion of lungsten and molybdenum!

\begin{tabular}{|c|c|c|c|c|c|c|c|}
\hline \multirow{2}{*}{$\begin{array}{c}\text { Days } \\
\text { following } \\
\text { treatment }\end{array}$} & \multirow[b]{2}{*}{ Item No. } & \multirow[b]{2}{*}{ Treatment } & \multicolumn{4}{|c|}{ Leaf enzymes (specific activity) } & \multirow[b]{2}{*}{ Leaf mean } \\
\hline & & & $\begin{array}{c}\beta-\text {-glycero- } \\
\text { phos- } \\
\text { phatase }\end{array}$ & ATP-ase & $\begin{array}{c}\text { Glucose- } \\
\text { 1-P } \\
\text { phos- } \\
\text { phatase }\end{array}$ & Amylase & \\
\hline \multirow[t]{4}{*}{0} & $\begin{array}{l}1 \\
2 \\
3 \\
4 \\
5\end{array}$ & $\begin{array}{l}\text { Control } \\
\text { W, } 1 \text { p.p.m. } \\
\text { W, } 10 \text { p.p.m. } \\
\text { W, } 100 \text { p.p.m. } \\
\text { W, } 200 \text { p.p.m. }\end{array}$ & $\begin{array}{l}30.1 \\
24.2 \\
23.4 \\
29.4 \\
27.2\end{array}$ & $\begin{array}{l}55.7 \\
43.6 \\
45.6 \\
56.0 \\
56.0\end{array}$ & $\begin{array}{r}10.0 \\
8.9 \\
7.8 \\
9.1 \\
9.9\end{array}$ & $\begin{array}{l}344 \\
300 \\
275 \\
335 \\
294\end{array}$ & $\begin{array}{r}109.9 \\
94.2 \\
88.2 \\
107.4 \\
96.8\end{array}$ \\
\hline & & W mean & 20.1 & 50.3 & 8.9 & 301 & 96.6 \\
\hline & $\begin{array}{l}6 \\
7 \\
8 \\
9\end{array}$ & $\begin{array}{l}\text { Mo, } 10 \text { p.p.m. } \\
\text { Mo, } 20 \text { p.p.m. } \\
\text { Mo, } 40 \text { p.p.m. } \\
\text { Mo, } 80 \text { p.p.m. }\end{array}$ & $\begin{array}{l}25.3 \\
27.6 \\
29.6 \\
30.6\end{array}$ & $\begin{array}{l}42.7 \\
52.3 \\
54.8 \\
58.6\end{array}$ & $\begin{array}{l}8.2 \\
7.7 \\
8.1 \\
9.2\end{array}$ & $\begin{array}{l}262 \\
272 \\
299 \\
294\end{array}$ & $\begin{array}{l}84.5 \\
89.9 \\
97.9 \\
98.1\end{array}$ \\
\hline & & Mo mean & 28.3 & 52.1 & 8.3 & 282 & 92.7 \\
\hline \multirow[t]{4}{*}{3} & $\begin{array}{l}10 \\
11 \\
12 \\
13 \\
14\end{array}$ & $\begin{array}{l}\text { Control } \\
\text { W, } 1 \text { p.p.m. } \\
\text { W, } 10 \text { p.p.m. } \\
\text { W, } 100 \text { p.p.m. } \\
\text { W, } 200 \text { p.p.m. }\end{array}$ & $\begin{array}{l}26.5 \\
30.2 \\
23.7 \\
34.7 \\
29.7\end{array}$ & $\begin{array}{l}48.5 \\
52.0 \\
40.9 \\
78.0 \\
59.6\end{array}$ & $\begin{array}{l}14.0 \\
13.9 \\
10.1 \\
12.7 \\
12.0\end{array}$ & $\begin{array}{l}247 \\
236 \\
190 \\
209 \\
258\end{array}$ & $\begin{array}{l}84.0 \\
83.0 \\
66.2 \\
83.6 \\
89.8\end{array}$ \\
\hline & & W mean & 29.6 & 57.6 & 12.2 & 223 & 80.6 \\
\hline & $\begin{array}{l}15 \\
16 \\
17 \\
18\end{array}$ & $\begin{array}{l}\text { Mo, } 10 \text { p.p.m. } \\
\text { Mo, } 20 \text { p.p.m. } \\
\text { Mo, } 40 \text { p.p.m. } \\
\text { Mo, } 80 \text { p.p.m. }\end{array}$ & $\begin{array}{l}32.5 \\
29.7 \\
40.3 \\
30.2\end{array}$ & $\begin{array}{l}63.6 \\
52.5 \\
86.9 \\
44.4\end{array}$ & $\begin{array}{r}10.8 \\
8.9 \\
11.7 \\
10.1\end{array}$ & $\begin{array}{l}258 \\
222 \\
274 \\
300\end{array}$ & $\begin{array}{r}91.2 \\
78.3 \\
103.2 \\
96.2\end{array}$ \\
\hline & & Mo mean & 33.2 & 61.9 & 10.4 & 264 & 92.3 \\
\hline \multirow[t]{4}{*}{$\mathbf{9}$} & $\begin{array}{l}19 \\
20 \\
21 \\
22 \\
23\end{array}$ & $\begin{array}{l}\text { Control } \\
\text { W, } 1 \text { p.p.m. } \\
\text { W, } 10 \text { p.p.m. } \\
\text { W, } 100 \text { p.p.m. } \\
\text { w, } 200 \text { p.p.m. }\end{array}$ & $\begin{array}{l}42.2 \\
34.6 \\
28.9 \\
37.5 \\
41.8\end{array}$ & $\begin{array}{l}60.8 \\
61.5 \\
58.5 \\
64.8 \\
70.0\end{array}$ & $\begin{array}{r}12.6 \\
10.9 \\
8.4 \\
11.1 \\
11.3\end{array}$ & $\begin{array}{l}486 \\
484 \\
287 \\
447 \\
502\end{array}$ & $\begin{array}{r}150.4 \\
147.8 \\
95.7 \\
140.1 \\
156.3\end{array}$ \\
\hline & & W mean & 35.7 & 63.7 & 10.4 & 430 & 134.9 \\
\hline & $\begin{array}{l}24 \\
25 \\
26 \\
27\end{array}$ & $\begin{array}{l}\text { Mo, } 10 \text { p.p.m. } \\
\text { Mo, } 20 \text { p.p.m. } \\
\text { Mo, } 40 \text { p.p.m. } \\
\text { Mo, } 80 \text { p.p.m. }\end{array}$ & $\begin{array}{l}32.4 \\
36.6 \\
27.9 \\
29.5\end{array}$ & $\begin{array}{l}55.5 \\
63.3 \\
55.0 \\
53.9\end{array}$ & $\begin{array}{l}9.3 \\
9.3 \\
6.4 \\
6.5\end{array}$ & $\begin{array}{l}290 \\
454 \\
319 \\
303\end{array}$ & $\begin{array}{r}96.8 \\
140.8 \\
102.1 \\
98.2\end{array}$ \\
\hline & & Mo mean & 31.6 & 56.9 & 7.9 & 342 & 109.6 \\
\hline
\end{tabular}


TABLE 3.-Conlinued

\begin{tabular}{|c|c|c|c|c|c|c|c|}
\hline \multirow{2}{*}{$\begin{array}{l}\text { Days } \\
\text { following } \\
\text { treatment }\end{array}$} & \multirow[b]{2}{*}{ Item no. } & \multirow[b]{2}{*}{ Treatment } & \multicolumn{4}{|c|}{ Leaf enzymes (specific activity) } & \multirow[b]{2}{*}{ Leaf mean } \\
\hline & & & $\begin{array}{c}\text { B-glycero- } \\
\text { phos- } \\
\text { phatase }\end{array}$ & ATP-ase & $\begin{array}{c}\text { Glucose- } \\
1-P \\
\text { phos- } \\
\text { phatase }\end{array}$ & Amylase & \\
\hline \multirow[t]{4}{*}{27} & $\begin{array}{l}28 \\
29 \\
30 \\
31 \\
32\end{array}$ & $\begin{array}{l}\text { Control } \\
\text { w, } 1 \text { p.p.m. } \\
\text { W, } 10 \text { p.p.m. } \\
\text { W, } 100 \text { p.p.m. } \\
\text { W, } 200 \text { p.p.m. }\end{array}$ & $\begin{array}{l}21.9 \\
37.0 \\
25.2 \\
34.1 \\
21.2\end{array}$ & $\begin{array}{l}32.3 \\
73.8 \\
46.2 \\
77.2 \\
41.8\end{array}$ & $\begin{array}{r}7.7 \\
10.3 \\
6.7 \\
7.8 \\
5.8\end{array}$ & $\begin{array}{l}140 \\
400 \\
203 \\
281 \\
187\end{array}$ & $\begin{array}{r}50.5 \\
130.3 \\
70.3 \\
100.0 \\
63.9\end{array}$ \\
\hline & & $\mathrm{W}$ mean & 29.4 & 59.8 & 7.7 & 208 & 91.2 \\
\hline & $\begin{array}{l}33 \\
34 \\
35 \\
36\end{array}$ & $\begin{array}{l}\text { Mo, } 10 \text { p.p.m. } \\
\text { Mo, } 20 \text { p.p.m. } \\
\text { Mo, } 40 \text { p.p.m. } \\
\text { Mo, } 80 \text { p.p.m. }\end{array}$ & $\begin{array}{l}34.6 \\
28.9 \\
27.0 \\
24.8\end{array}$ & $\begin{array}{l}66.9 \\
57.7 \\
52.6 \\
48.2\end{array}$ & $\begin{array}{l}7.1 \\
5.3 \\
7.3 \\
6.0\end{array}$ & $\begin{array}{l}359 \\
271 \\
263 \\
220\end{array}$ & $\begin{array}{r}116.9 \\
90.7 \\
87.5 \\
74.8\end{array}$ \\
\hline & & Mo mean & 28.8 & 56.4 & 6.4 & 278 & 92.4 \\
\hline \multicolumn{2}{|c|}{ Grand mean for: } & $\begin{array}{l}\text { Control } \\
\text { W } \\
\text { Mo }\end{array}$ & $\begin{array}{l}32.7 \\
30.2 \\
30.5\end{array}$ & $\begin{array}{l}49.3 \\
57.9 \\
56.8\end{array}$ & $\begin{array}{r}11.1 \\
9.8 \\
8.3\end{array}$ & $\begin{array}{l}304 \\
307 \\
292\end{array}$ & $\begin{array}{r}99.3 \\
101.2 \\
96.9\end{array}$ \\
\hline
\end{tabular}

1 Each figure represents the computed mean of 3 replicates.

stimulated by $M o$ and $W$ (table 3 , items 13, 14, 15, 17, 23, and 29-36; table 4, items 13, 20-27, 32, 33, 34, and 35).

The $\mathrm{W}$ treatment of 10 p.p.m., which caused the greatest sucrose increases, also effected the most consistent $W$ suppression of G-1-P ase. This agrees well with earlier conclusions that G-1-P is a decisive factor in low phosphatase-high sucrose relationships $(1,2,8)$. However, Mo was more effective than $W$ in suppressing phosphatase, yet was not so successful as $\mathrm{W}$ in promoting sucrose.

Anylase was generally inhibited by $W$ and Mo at 3 and 9 days, but appeared to be stimulated by both elements at 27 days. The 27 -day response is again misleading, since it was the control group rather than treatments which produced the greatest enzyme change. This point will be discussed later.

A glance at the pretreatment enzyme data (tables 3 and 4 , "0 days") tells us that unknown factors were already affecting the respective groups when the sprays were applied. It has been pointed out that some sucrose variations existed between control and treatment plants at " 0 days" (table 2), and it is evident that a suppression of phosphatases and amylase 
TABLE 4.-Mean values for merislem enzymes of immalure sugarcane following foliar application of tungsten and molybdenum ${ }^{1}$

\begin{tabular}{|c|c|c|c|c|c|c|c|c|}
\hline \multirow{3}{*}{$\begin{array}{c}\text { Days } \\
\text { following } \\
\text { treat- } \\
\text { ment }\end{array}$} & \multirow{3}{*}{$\begin{array}{l}\text { Item } \\
\text { no. }\end{array}$} & \multirow{3}{*}{ Treatment } & \multicolumn{5}{|c|}{ Meristem enzymes (specific activity) } & \multirow{3}{*}{$\begin{array}{c}\text { Meristem } \\
\text { mean }\end{array}$} \\
\hline & & & \multirow{2}{*}{$\begin{array}{c}\beta- \\
\text { glycero- } \\
\text { phos- } \\
\text { phatase }\end{array}$} & \multicolumn{4}{|c|}{ Glucose-1-P } & \\
\hline & & & & ATP-ase & $\begin{array}{c}\text { Phos- } \\
\text { phatase }\end{array}$ & Amylase & Invertase & \\
\hline \multirow[t]{4}{*}{0} & $\begin{array}{l}1 \\
2 \\
3 \\
4 \\
5\end{array}$ & $\begin{array}{l}\text { Control } \\
\text { W, } 1 \text { p.p.m. } \\
\text { W, } 10 \text { p.p.m. } \\
\text { W, } 100 \text { p.p.m. } \\
\text { W, } 200 \text { p.p.m. }\end{array}$ & $\begin{array}{l}27.6 \\
28.4 \\
29.1 \\
31.7 \\
27.8\end{array}$ & $\begin{array}{l}26.4 \\
30.3 \\
25.7 \\
29.8 \\
24.5\end{array}$ & $\begin{array}{l}8.0 \\
8.4 \\
6.5 \\
7.7 \\
7.9\end{array}$ & $\begin{array}{l}145 \\
137 \\
120 \\
157 \\
145\end{array}$ & $\begin{array}{l}20.0 \\
25.4 \\
19.6 \\
28.0 \\
25.2\end{array}$ & $\begin{array}{l}45.4 \\
45.9 \\
40.2 \\
50.8 \\
46.1\end{array}$ \\
\hline & & W mean & 29.3 & 27.6 & 7.6 & 139 & 24.6 & 45.6 \\
\hline & $\begin{array}{l}6 \\
7 \\
8 \\
9\end{array}$ & $\begin{array}{l}\text { Mo, } 10 \text { p.p.m. } \\
\text { Mo, } 20 \text { p.p.m. } \\
\text { Mo, } 40 \text { p.p.m. } \\
\text { Mo, } 80 \text { p.p.m. }\end{array}$ & $\begin{array}{l}26.0 \\
24.0 \\
28.8 \\
31.8\end{array}$ & $\begin{array}{l}24.8 \\
21.5 \\
28.1 \\
31.2\end{array}$ & $\begin{array}{l}6.5 \\
6.5 \\
6.7 \\
9.0\end{array}$ & $\begin{array}{l}135 \\
129 \\
149 \\
178\end{array}$ & $\begin{array}{l}21.8 \\
22.5 \\
27.9 \\
18.0\end{array}$ & $\begin{array}{l}43.0 \\
40.7 \\
48.1 \\
53.6\end{array}$ \\
\hline & & Mo mean & 27.9 & 26.4 & 7.2 & 148 & 22.6 & 46.4 \\
\hline \multirow[t]{4}{*}{3} & $\begin{array}{l}10 \\
11 \\
12 \\
13 \\
14\end{array}$ & $\begin{array}{l}\text { Control } \\
\text { W, } 1 \text { p.p.m. } \\
\text { W, } 10 \text { p.p.m. } \\
\text { W, } 100 \text { p.p.m. } \\
\text { W, } 200 \text { p.p.m. }\end{array}$ & $\begin{array}{l}36.6 \\
25.7 \\
34.2 \\
50.4 \\
38.2\end{array}$ & $\begin{array}{l}43.6 \\
30.5 \\
41.9 \\
70.2 \\
46.1\end{array}$ & $\begin{array}{r}9.6 \\
7.4 \\
8.4 \\
13.1 \\
8.0\end{array}$ & $\begin{array}{r}107 \\
82 \\
98 \\
130 \\
113\end{array}$ & $\begin{array}{l}26.1 \\
19.8 \\
29.4 \\
32.5 \\
21.1\end{array}$ & $\begin{array}{l}44.6 \\
33.1 \\
42.4 \\
59.2 \\
45.3\end{array}$ \\
\hline & & W mean & 37.1 & 47.2 & 9.2 & 106 & 25.7 & 45.0 \\
\hline & $\begin{array}{l}15 \\
16 \\
17 \\
18\end{array}$ & $\begin{array}{l}\text { Mo, } 10 \text { p.p.m. } \\
\text { Mo, } 20 \text { p.p.m. } \\
\text { Mo, } 40 \text { p.p.m. } \\
\text { Mo, } 80 \text { p.p.m. }\end{array}$ & $\begin{array}{l}31.3 \\
30.7 \\
33.4 \\
25.7\end{array}$ & $\begin{array}{l}34.5 \\
37.7 \\
39.8 \\
41.4\end{array}$ & $\begin{array}{l}8.6 \\
6.9 \\
7.4 \\
7.5\end{array}$ & $\begin{array}{l}91 \\
92 \\
99 \\
89\end{array}$ & $\begin{array}{l}28.3 \\
22.9 \\
28.9 \\
20.2\end{array}$ & $\begin{array}{l}38.7 \\
38.0 \\
41.7 \\
36.8\end{array}$ \\
\hline & & Mo mean & 30.3 & 38.4 & 7.6 & 92.8 & 25.1 & 38.8 \\
\hline \multirow[t]{4}{*}{9} & $\begin{array}{l}19 \\
20 \\
21 \\
22 \\
23\end{array}$ & $\begin{array}{l}\text { Control } \\
\text { w, } 1 \text { p.p.m. } \\
\text { W, } 10 \text { p.p.m. } \\
\text { w, } 100 \text { p.p.m. } \\
\text { w, } 200 \text { p.p.m. }\end{array}$ & $\begin{array}{l}30.5 \\
30.8 \\
34.5 \\
40.3 \\
51.0\end{array}$ & $\begin{array}{l}29.6 \\
36.6 \\
44.9 \\
48.6 \\
79.5\end{array}$ & $\begin{array}{r}9.9 \\
8.1 \\
7.6 \\
8.6 \\
11.5\end{array}$ & $\begin{array}{l}127 \\
124 \\
135 \\
134 \\
167\end{array}$ & $\begin{array}{l}31.4 \\
23.5 \\
17.0 \\
28.3 \\
21.8\end{array}$ & $\begin{array}{l}45.7 \\
44.6 \\
47.8 \\
51.9 \\
66.2\end{array}$ \\
\hline & & $W$ mean & 39.2 & 52.4 & 8.9 & 140 & 22.7 & 52.6 \\
\hline & $\begin{array}{l}24 \\
25 \\
26 \\
27\end{array}$ & $\begin{array}{l}\text { Mo, } 10 \text { p.p.m. } \\
\text { Mo, } 20 \text { p.p.m. } \\
\text { Mo, } 40 \text { p.p.m. } \\
\text { Mo, } 80 \text { p.p.m. }\end{array}$ & $\begin{array}{l}37.8 \\
31.1 \\
36.0 \\
30.6\end{array}$ & $\begin{array}{l}44.8 \\
40.5 \\
53.6 \\
39.0\end{array}$ & $\begin{array}{l}5.4 \\
4.4 \\
4.7 \\
5.1\end{array}$ & $\begin{array}{l}132 \\
113 \\
133 \\
122\end{array}$ & $\begin{array}{l}23.7 \\
21.9 \\
20.5 \\
18.7\end{array}$ & $\begin{array}{l}48.7 \\
42.2 \\
49.6 \\
43.1\end{array}$ \\
\hline & & Mo mean & 33.9 & 44.5 & 4.9 & 125 & 21.2 & 45.9 \\
\hline
\end{tabular}


TABLE 4.--Continued

\begin{tabular}{|c|c|c|c|c|c|c|c|c|}
\hline \multirow{3}{*}{$\begin{array}{c}\text { Days } \\
\text { following } \\
\text { treat- } \\
\text { ment }\end{array}$} & \multirow{3}{*}{$\begin{array}{l}\text { Item } \\
\text { no. }\end{array}$} & \multirow{3}{*}{ Treatment } & \multicolumn{5}{|c|}{ Meristem enzymes (specific activity) } & \multirow{3}{*}{$\begin{array}{l}\text { Meristern } \\
\text { mean }\end{array}$} \\
\hline & & & \multirow{2}{*}{$\begin{array}{c}\begin{array}{c}\beta- \\
\text { glycero- } \\
\text { phos } \\
\text { phatase }\end{array}\end{array}$} & \multicolumn{4}{|c|}{ Glucose-1-P } & \\
\hline & & & & ATP-ase & $\begin{array}{l}\text { Phos- } \\
\text { phatase }\end{array}$ & Amylase & Invertase & \\
\hline \multirow[t]{11}{*}{27} & 28 & Control & 19.9 & 22.8 & 9.6 & 89 & 15.2 & 31.3 \\
\hline & 29 & W, 1 p.p.m. & 23.2 & 24.3 & 10.1 & 80 & 16.5 & 32.0 \\
\hline & 30 & W, 10 p.p.m. & 21.9 & 23.3 & 7.9 & 79 & 13.8 & 29.2 \\
\hline & 31 & W, 100 p.p.m. & 22.9 & 26.8 & 7.9 & 88 & 15.0 & 32.1 \\
\hline & 32 & W, 200 p.p.m. & 25.9 & 32.9 & 9.5 & 85 & 15.8 & 33.8 \\
\hline & & $\mathrm{W}$ mean & 23.5 & 26.8 & 8.9 & 84.5 & 15.3 & 31.8 \\
\hline & 33 & Mo, 10 p.p.m. & 27.5 & 36.1 & 8.6 & 96 & 16.2 & 36.9 \\
\hline & 34 & Mo, 20 p.p.m. & 25.1 & 29.3 & 6.9 & 77 & 17.6 & 31.2 \\
\hline & 35 & Mo, 40 p.p.m. & 25.2 & 28.7 & 6.5 & 91 & 16.7 & 33.6 \\
\hline & 30 & Mo, 80 p.p.m. & 21.3 & 23.0 & 8.1 & 79 & 15.7 & 29.4 \\
\hline & & Mo mean & 24.8 & 29.3 & 7.5 & 86 & 16.6 & 32.8 \\
\hline \multirow{4}{*}{\multicolumn{2}{|c|}{$\begin{array}{l}\text { Girasd mean } \\
\text { for: }\end{array}$}} & & & & & & & \\
\hline & & Control & 28.7 & 30.6 & 9.3 & 117 & 23.2 & 41.8 \\
\hline & & & 32.3 & 38.5 & 8.7 & 117 & 22.1 & 43.7 \\
\hline & & Mo & 29.2 & 34.7 & 6.8 & 113 & 21.4 & 41.0 \\
\hline
\end{tabular}

${ }^{1}$ Each figure represents the computed mean of 3 replicates.

was already in effect in most plants where sucrose was high. In this instance the $\beta$-glycerophosphatase and ATP-ase systems were suppressed, as well as G-1-P ase. Whatever means later enabled Mo and W to stimulate phosphatase were not applicable to G-1-P ase, which again points to the critical position of this compound in the overall scheme of sucrose production.

A careful study of meristem sugar and enzyme data shows that invertase was probably more responsible for sucrose fluctuations in that tissue than was G-1-P ase. For example, meristem sucrose declined sharply at 9 days (table 2, item 19), even though G-1-P ase was no more active than it had been at 3 days (table 4 , item 10), or would be at 27 days (table 4 , item 28). Invertase, on the other hand, achieved its highest level of the study at 9 days (table 4, item 19). The only other invertase response to approach the control value appeared among plants receiving 100 p.p.m. of $\mathrm{W}$, and this, in turn, coincides with the second lowest meristem sucrose level found at the 9-day harvest (table 2, item 22). Control invertase declined by about 50 percent between the 9 - and 27 -day harvests. Sucrose more than doubled during this period (table 2, item 19 vs. item 28). 
It should be noted that total ketose values were considerably higher for meristem than for leaves, and that fructose rather than sucrose was the dominant ketose of meristem. This agrees with earliel observations $(4,8)$ and probably reflects the predominance of invertase in meristem, as compared to leaves.

We have found, as a rule, that total ketose values seldom vary greatly, and that the appearance of high sucrose is usually mirrored by a decline of fructose. In one instance, high sucrose concentrations induced by nitrate shortages caused almost total depletion of fructose (1). One implication is that hexose synthesis is not in itself a very limiting factor in determining sucrose level. Rather, sucrose content must be decided by reactions involving fructose and glucose already formed, and which may enter into metabolic pathways, pathways of sucrose formation, starch formation, direct oxidation, and so on. This again accounts for the observed relations between sucrose level and the phosphatases, amylase, and invertase, each of which catalyzes reactions very close to the final steps of sucrose synthesis.

Another implication of a constant ketose-variable sucrose system is that fructose is not a limiting factor, and that by the reaction

$$
\begin{gathered}
\text { G-1-P }+ \text { Fructose } \underset{\text { or }}{\rightleftharpoons} \text { Sucrose }+\mathrm{H}_{3} \mathrm{PO}_{4}, \\
\text { G-1-P + UTP } \underset{\text { UDPG }+ \text { UDP, }}{\rightleftharpoons}, \\
\text { UDPG + Fructose } \rightleftharpoons \text { Sucrose + UDP, }
\end{gathered}
$$

enough fructose is available to match whatever G-1-P or UDPG is able to enter the sucrose-forming pathways. This again points to the critical nature of G-1-P, and helps to explain the very consistent suppression of G-1-P ase by treatments which increase sucrose $(1,3,2,8)$.

\section{SIGNIFICANCE OF IN VIVO SUCROSE ENZYME RELATIONSHIPS}

Aside from the fact that Mo and $\mathrm{W}$ can inhibit enzymes, and at least temporarily increase sucrose, several treatment effects gave important leads as to biochemical consequences of applied enzyme inhibitors. In particular, the stimulation of ATP-ase by Mo and W, and the alteration of "normal" enzyme fluctuations, merit careful consideration.

In vitro, the ATP-ase of cane is about 80 percent inhibited by $6 \times 10^{-3}$ $\mu$ mole of Mo or W per milliliter of digest (5). Thus there was no reason to expect an in vivo stimulation of ATP-ase by these elements, although this in fact did occur in leaves (table 3, items 13, 14, 15, 17, 23, and 29-38) and in meristem (table 4, items 13, 21, 22, 23, 24, 25, 26, 27, 32, and 33). The importance of a stinulated ATP-ase reaction can hardly be overemphasized. As a recipient of energy from exergonic reactions, ATP serves 
as a source of the energy which drives endergonic processes of life ${ }^{5}$, and enzymes catalyzing its degradation thereby assume considerable importance.

It is felt that those $\mathrm{W}$ treatments which stimulated ATP-ase did so by shifting the plant into a new level of biochemical activity, rather than by any direct effects upon ATP-ase. In such a physiological state the apparently stimulated enzyme may have been relatively as inactive as that of control plants, or perhaps even less active in a relative sense. To support this contention it is noted that whereas W increased ATP-ase as a main effect, those treatments which actually caused sucrose increases generally left ATP-ase activity at a lower level than those which did not increase sucrose. ${ }^{6}$ The means of Mo action are less clear. Possibly Mo acted upon another enzyme associated with the adenylic acid system, such as one affecting the balance between the high-energy phosphate of ATP and that of a storage form such as creatin phosphate. An usually high level of ATP might then induce a greater synthesis of ATP-ase.

While studying the effects of applied indole-3-acetjc acid and 2,4-dichlorophenoxyacetic acid, we observed what appeared to be a stimulation of treated cane into a greater state of biochemical activity, much like that of the W and ATP-ase relationship. The growth-regulator effects were apparent upon many enzymes, and to some extent had been expected. However, we had postulated that Mo and W would specifically inhibit phosphatase, or at the most inhibit the hydrolytic group composed of phosphatase, amylase, and invertase. By having caused the stimulation of the key enzyme of the adenylic acid system, whether directly or indirectly, Mo and $W$ have shown far greater depth of potential than was anticipated. In this capacity alone they may eventually be shown to have growthregulating powers.

Another unexpected and perhaps more significant result of Mo and W treatments was the fact that they prevented treated plants from following enzyme fluctuations experienced by the control group. For example, at 27 days the $W$ treatments of 1 and 100 p.p.m. appeared to cause striking stimulation of leaf ATP-ase and amylase (table 3, items 29 and 31). What actually occurred was a pronounced shift downward in enzyme activity of control plants. The activity of enzymes from treated plants remained closer to the 9-day levels. Had only the 27-day harvest been taken, these enzymes surely would have been credited with being profoundly stimulated.

\footnotetext{
s The adenylic acid system and the importance of ATP-ase in sugarcane have been discussed in detail in an earlier report $(1)$.

- As examples, compare leaf sucrose (table 2, items 29 and 31 vs. items 30 and 32) with leaf ATP-ase (table 3, items 29 and 31 vs. items 30 and 32). Also compare leaf sucrose (table 2, items 11 vs. 12 and 13 vs. 14) with leaf ATP-ase (table 3, items 11 vs. 12 and 13 vs. 14).
} 
The same principle was demonstrated in meristem at 9 days. For some physiological reason the control plants experienced a sharp sucrose decline, which, as pointed out earlier, was apparently mediated through an increased invertase action (table 2, item 19; table 4, item 19). None of the plants receiving Mo and $\mathrm{W}$ experienced so great an invertase increase, and all were credited with promoting sucrose. We can only guess as to the stimulus for increased invertase action-perhaps there was a temporary need for more free hexose. Yet, whatever the stimulus, treated plants did not answer the call. This is not a negative treatment response. Rather, Mo and $W$ were exhibiting a far greater capacity to influence enzymes than our original hypothesis had foreseen.

Numerous physiological changes can occur in sugarcane before it is milled, some of which tend to deplete the eventual sugar yield. If, for example, enzyme-regulators could be found to retard the stimulus for flowering, or the biochemical changes responsible for sucrose decline after harvest, these could conceivably increase sugar yield more cheaply and effectively than any treatment now known. The processes of lodging, inhibited tillering, and excessive transpiration must be initiated by biochemical stimuli of considerable magnitude. Enzyme-regulators might delay, retard, or even eliminate such stimuli. In those instances where climatic and nutritional factors provide the stimulus for undesirable changes, key enzymes might be altered just sufficiently so that such factors cannot cause major changes. These suggestions may seem to verge upon fantasy, yet the only fantastic feature about them is the amount of research required to discover such materials, their effective concentrations, and the proper time and method for their application. The principle itself is becoming increasingly logical.

\section{SUMMARY}

Variable concentrations of tungsten and molybdenum were applied as foliar sprays to 12-week-old sugarcane grown in sand culture. The objectives were to evaluate both elements as in vivo inhibitors of amylase, invertase, and acid phosphatases, and to test further the hypothesis that such inhibition will result in increased sucrose content. Leaf and meristem samples were harvested just prior to initial treatment, and at 3,9 , and 27 days thereafter.

Glucose-1-phosphatase and amylase were generally inhibited by both elements, and increased sucrose usually appeared as a consequence. Tungsten at 10 p.p.m. significantly increased sucrose in leaf and meristem at all posttreatment harvests. Molybdenum was a more effective phosphatase inhibitor than tungsten, but was less effective in promoting sucrose production. Molybdenum increased sucrose in meristem only. 
$\beta$-glycerophosphatase and ATP-ase were stimulated by some of the treatments. This was totally unexpected since both enzymes are known to be inhibited by tungsten and molybdenum in vitro. Certain treatments appeared to reduce the enzymes' capacity to experience major fluctuations. The significance of this property and the stimulation of ATP-ase are discussed at length.

\section{RESUMEN}

Se aplicaron tungsteno y molibdeno en concentraciones variables y en forma de aspersión, a caña de azúcar de 12 semanas de edad sembrada en arena. Los objetivos fueron evaluar ambos agentes inhibitorios de la amilasa, la invertasa y las fosfatasas ácidas, y continuar probando la hipótesis de que tal inhibición producirá un aumento en el contenido de la sacarosa. Se tomaron muestras de las hojas y del meristemo inmediatamente antes del tratamiento inicial, y 3,9 y 27 días después.

Por lo general, ambos elementos actuaron como inhibidores de la fosfatasa de glucosa-1 y la amilasa, produciéndose casi siempre, como resultado, un aumento en la sacarosa. El tungsteno, a una concentración de $10 \mathrm{ppm}$., aumentó significativamente el contenido de sacarosa en la hoja y en el meristemo de todas las cosechas que se hicieron después de terminados los tratamientos.

Algunos de los tratamientos estimularon la $\beta$-glicerofosfatasa y la ATPasa. Tal reacción no se esperaba, porque se sabe que el tungsteno y el molibdeno actúan como inhibidores de ambas enzimas in vitro. Ciertos tratamientos aparentemente disminuyeron la capacidad de las enzimas para tolerar grandes fluctuaciones. Se discuten detalladamente el significado de esta propiedad y el estímulo de la ATP-asa.

\section{LITERATURE CITED}

1. Alexander, A. G., Sucrose-enzyme relationships in immature sugarcane as affected by varying levels of nitrate and potassium supplied in sand culture, $J$. Agr. Univ. P.R. 48 (3): 165-231, 1964.

2. - Induction of varying sugar levels in leaves of immature sugarcane by use of acid phosphatase inhibitors, J. Agr. Univ. P.R. 49 (1): 35-59, 1965.

3 . - Changes in leaf-sugar content and enzyme activity of immature sugarcane following foliar application of indole-3-acetic acid, 2,4-dichlorophenoxyacetic acid, and maleic hydrazide, J. Agr. Univ. P.R. 49 (1): 1-34, 1965.

4. - Behavior of enzymes governing starch and sucrose-forming pathways in two sugarcane varieties supplied with variable nitrate and phosphate in sand culture, J. Agr. Univ. P.R. 49 (2): 153-75, 1965.

5. - - Hydrolytic proteins of sugarcane: The acid phosphatases, J. Agr. Univ. P.R. 49 (2): 204-28, 1965.

6. - The hydrolytic proteins of sugarcane: The acid invertases, J. Agr. Univ. P.R. 49 (3) : 287-307, 1965. 


\section{JOURNAL OF AGRICUITURE OF UNIVERSITY OF PUERTO RICO}

7. - Hydrolytic proteins of sugarcane: Amylase, J. Agr. Univ. P.R. 49 (3): 308$24,1965$.

8. - Sucrose-enzyme relationships in immature sugarcane treated with variable molybdenum, calcium, iron, boron, lead, trichloroacetic acid, $\beta$-glycerophosphate, and starch, J. Agr. Univ. P.R. 49 (4): 443-61, 1965.

9. Cardini, C. E., Leloir, L. F., and Chiriboga, J., The biosynthesis of sucrose, $J$. Biol. Chem. 214: 149-55, 1955.

10. Roe, J. R., A colorimetric method for the determination of fructose in blood and urine, J. Biol. Chem. 107: 15-22, 1934. 